\title{
Coffee consumption and risk of coronary heart disease: A meta-analysis
}

\author{
Francesco Sofi ${ }^{\mathrm{a}, \mathrm{d}, *}$, Andrea A. Conti ${ }^{\mathrm{a}, \mathrm{b}}$, Anna Maria Gori ${ }^{\mathrm{a}, \mathrm{d}}$, \\ Maria Luisa Eliana Luisi ${ }^{\mathrm{b}}$, Alessandro Casini ${ }^{\mathrm{c}, \mathrm{d}}$, \\ Rosanna Abbate ${ }^{a, d}$, Gian Franco Gensini ${ }^{a, b}$
}

\author{
${ }^{a}$ Department of Medical and Surgical Critical Care and Center for the Study at \\ Molecular and Clinical Level of Chronic, Degenerative and Neoplastic Diseases \\ to Develop Novel Therapies, University of Florence, Florence, Italy \\ ${ }^{\mathrm{b}}$ Don Carlo Gnocchi Foundation, IRCCS, Florence, Italy \\ c Department of Clinical Pathophysiology, Unit of Clinical Nutrition, \\ University of Florence, Florence, Italy \\ ${ }^{d}$ Centro Interdipartimentale di Ricerca per la Valorizzazione degli Alimenti (CeRA), \\ University of Florence, Florence, Italy
}

Received 27 May 2006; received in revised form 14 July 2006; accepted 22 July 2006

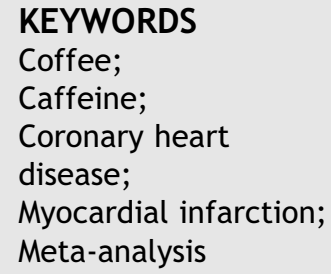

\begin{abstract}
Background and aims: During the past three decades the relationship between habitual coffee drinking and coronary heart disease (CHD) has been assessed in numerous studies, with conflicting results. The aim of this study was to systematically examine the data published on the association between habitual coffee consumption and risk of CHD.

Methods and results: Thirteen case-control and 10 cohort studies were included. Case-control studies incorporated 9487 cases of CHD and 27,747 controls, and cohort studies included a total of 403,631 participants that were followed for between 3 and 44 years. The summary of odds ratios (OR) for the case-control studies showed statistically significant associations between coffee consumption and CHD for the highest intake group (>4 cups/day), OR 1.83 (95\% Cl 1.49-2.24; $P<0.0001)$, and for the second highest category (3-4 cups/day), OR $1.33(95 \% \mathrm{Cl}$ $1.04-1.71 ; P<0.0001)$, while no significant association emerged for low daily coffee intake ( $\leq 2$ cups/day), OR 1.03 (95\% Cl 0.87-1.21; $P=0.45)$. The analysis of long-term follow-up cohort studies did not show any association between the consumption of coffee and CHD, with a relative risk (RR) of $1.16(95 \% \mathrm{Cl} 0.95-1.41$; $P=0.14)$ for the highest category, and $1.05(95 \% \mathrm{Cl} 0.90-1.22 ; P=0.57)$ and
\end{abstract}

* Corresponding author. Department of Medical and Surgical Critical Care, Thrombosis Centre, University of Florence, Azienda Ospedaliero-Universitaria Careggi, Viale Morgagni 85, 50134 Florence, Italy. Tel.: +39 055 7949420; fax: +39 0557949418.

E-mail address: francescosofi@gmail.com (F. Sofi). 
$1.04(95 \% \mathrm{Cl} 0.90-1.19 ; P=0.60)$ for the second and third highest categories, respectively. These results did not differ substantially when controlling for region of origin, fatal and non-fatal events, year of publication, and number of years of follow-up.

Conclusions: Despite a significant association between high consumption of coffee and $\mathrm{CHD}$ reported among case-control studies, no significant association between daily coffee consumption and CHD emerged from long-term follow-up prospective cohort studies.

(c) 2006 Elsevier B.V. All rights reserved.

\section{Introduction}

Coffee is one of the most widely consumed beverages in the world. Since early 1970s, coffee consumption has attracted interest as a potential health hazard due to the positive association with coronary heart disease (CHD) reported in a number of case-control and cohort studies [1-14]. However, in contrast with these results, other case-control and cohort studies performed in different population groups showed negative results, perhaps indicating that in some of the previous studies the strong association reported between coffee consumption and CHD resulted from inappropriate statistical analysis, i.e. lack of adjustment for possible confounders [15-28].

To date, the question whether coffee drinking increases the risk of CHD continues to be controversial. Meta-analyses of the association between coffee consumption and CHD carried out in the 1990s reported conflicting results within the different types of studies analysed, as shown by the positive association with CHD found among case-control but not among cohort studies. In 1992, Myers and Babinski carried out a metaanalysis of cohort prospective studies and reported no influence of coffee on CHD [29]; whereas in 1993, Greenland reported a positive association with CHD among case-control studies, but not among prospective cohort studies [30]. The latter association is supported by the most recent analysis conducted in 1994 by Kawachi et al., which showed an increased risk of CHD with consumption of five cups of coffee per day, but only among case-control studies [31].

Currently, the influence of coffee on the health of people, is considered of great significance due to the enormous consumption worldwide. How ever, analysing the occurrence of disease in relation to intake of coffee is difficult, mainly due to the complexities that arise when trying to define a coffee beverage. Coffee, as a beverage made from the extract of coffee beans, is available in a remarkable variety of different types throughout the world, in terms of brewing method and caffeine content; even within the same country methods of preparation and drinking preferences differ significantly. This could partly explain the contrasting results reported in the literature. Hence, we decided to conduct a systematic review with meta-analysis of the available epidemiological studies, both case-control and prospective, in order to evaluate if habitual coffee consumption is associated with an increased risk of CHD.

\section{Methods}

\section{Study selection}

We conducted a systematic literature search of MEDLINE from 1966, EMBASE from 1990, Science Citation Index from 1994, and the Cochrane Systematic Review Database up to April 2006, to identify studies that examined the association between habitual coffee consumption and CHD, with an analytical design-either case-control (retrospective or nested) or longitudinal. We used a combined text word and medical subject headings (MeSH) search strategy with the terms 'coffee' and 'caffeine' in combination with 'cardiovascular diseases', 'coronary artery disease', 'coronary heart disease', 'myocardial infarction', and 'acute coronary syndromes'. We included references listed in the retrieved articles as well as in review articles and in meta-analyses. The aim of this study was to evaluate the possible association between coffee consumption and occurrence of $\mathrm{CHD}$, with non-users or the lowest category as a reference. Hence, to be included in the meta-analysis, studies had to provide, as primary or secondary outcome, the association between coffee and CHD, as well as estimates of associations for the different categories of coffee consumption, and full information on study design and participant characteristics.

Our search strategy generated 24 potentially relevant case-control articles $[1,2,5,7-12,21-23$, 
26,32-42]. Four studies were excluded since they did not contain sufficient information to enable an estimate of risk or its standard error to be calculated for all categories of coffee consumption $[1,33-35]$. Five studies were excluded because they did not report outcomes of interest for this meta-analysis [36-40], one because it reported only data for decaffeinated coffee consumption [41], and another study [8] represented the final data from a previous paper [7], so only the final paper was included. Hence, 13 case-control papers were included in the final analysis $[2,5,8-$ $12,21-23,26,32,42]$, incorporating 9487 cases of CHD and 27,747 controls. With regard to cohort prospective studies, we identified 38 potentially relevant studies $[3,4,6,13-20,24,25,27,28,33$, 43-64], of which only 10 satisfied the inclusion criteria $[4,14,17,20,24,25,27,28,43]$. Seventeen studies contained insufficient data to enable the associations between different categories of coffee consumption and CHD to be estimated $[3,6,15,19,33,44-55]$. A further six studies reported outcomes that were not relevant to the present study [56-61]. In addition, the papers by Martin et al. [13] and Hakim et al. [62] were excluded since they studied a population of hypertensives, and two further studies $[63,64]$ were not included in this review because they included a category other than that of very low consumption as a reference. Finally, papers by Willett et al. [18] and by Grobbee et al. [16] were replaced by the work of Lopez-Garcia et al. [27], which included updated follow-up analyses deriving from the former two preliminary studies.

\section{Data extraction}

All data were independently extracted by two investigators (F.S. and A.M.G.) through use of a standardized data extraction tool, and were entered into separate databases. Data regarding study design, participant characteristics, country of origin, adjustment for potential confounders, measurements of coffee consumption, outcomes, and estimates of associations, were sought from each article. Results were compared, and disagreements were resolved by consensus. In cases in which there was more than one published report on the same population or group of patients, the most recent article was selected for analysis.

\section{Statistical analysis}

Data were analysed by using Review Manager (RevMan) software for Windows (version 4.2) by the Cochrane Collaboration, 2003, and STATA (version 8.2). We distinguished four levels of coffee consumption: the highest category of consumption (mainly $>4$ cups per day, except for Tavani et al. [32] and Kalandidi et al. [10] among case-control studies, and Lindsted et al. [14] among cohort studies, where $>3$ cups per day was used); the second highest category of coffee consumption (mainly 3-4 cups per day, excluding Jick et al. [2] and Rosenberg et al. [8] among case-control studies, and papers by Klatsky and colleagues [24,43] among cohort studies, where a range between $1-5$ cups per day was used); the third highest category of coffee consumption (mainly $\leq 2$ cups per day); and the reference category (none or $<1$ cup per day). Studies not reporting categories of coffee consumption were excluded from each analysis to improve comparability with other studies. Thus, among casecontrol studies, three studies from the analysis of the second highest category $[10,21,22]$ and two from the analysis of the third category [2,9] were excluded. Similarly, as for cohort prospective studies, two studies from the comparison of the second highest category $[14,17]$ and one study from the analysis of the third category [17] were excluded. We used the results of the original studies from multivariable models with the most complete adjustment for potential confounders; the confounding variables included in this analysis are shown in Tables 1 and 2 . The analysis was carried out using a random-effects model that accounts for interstudy variation and provides a more conservative estimate than a fixed model. Thus, we calculated random summary odds ratios (OR) and relative risks (RR) with 95\% confidence intervals $(\mathrm{Cl})$ for case-control and cohort studies, respectively, by using the inverse-variance method. The potential sources of heterogeneity were assessed using the standard chi-square test. In addition, the statistic $I^{2}$ was used to investigate heterogeneity by examining the extent of inconsistency across the study results. To examine the source of heterogeneity across studies we conducted sensitivity analyses, according to some characteristics of the studies included in the meta-analysis; i.e. the different region of origin of the studies (Europe/USA), the year of publication (studies published before and after the median year of publication of all the studies combined, i.e. 1995), as well as outcome measures (fatal, non-fatal events), and number of years of follow-up within cohort studies (before and after the median years of follow-up of all the studies combined, i.e. 15 years).

Funnel plots were performed to assess potential publication bias, as suggested by Egger et al. [65]. Moreover, to estimate whether publication bias 
Table 1 Characteristics of case-control studies included in the meta-analysis

\begin{tabular}{|c|c|c|c|c|c|c|c|c|}
\hline Source, year & Country & $\begin{array}{l}\text { Cases/ } \\
\text { controls }\end{array}$ & $\begin{array}{l}\text { Age } \\
\text { (years) }\end{array}$ & Gender & Outcome & $\begin{array}{l}\text { Coffee } \\
\text { consumption }\end{array}$ & $\begin{array}{l}\text { Adjusted OR } \\
(95 \% \mathrm{Cl})\end{array}$ & Adjustment \\
\hline Jick et al. 1973 [2] & USA & $440 / 12319$ & $40-69$ & $M / F$ & MI & $\begin{array}{l}\text { None } \\
1-5 \mathrm{cups} / \text { day } \\
\geq 6 \mathrm{cups} / \text { day }\end{array}$ & $\begin{array}{l}1.00 \\
1.6(1.3-2.0) \\
2.2(1.6-3.1)\end{array}$ & $\begin{array}{l}\text { Age, gender, } \\
\text { history of MI }\end{array}$ \\
\hline $\begin{array}{l}\text { Rosenberg et al. } \\
1987 \text { [8] (final data } \\
\text { of Rosenberg } \\
\text { et al. } 1980 \text { [7]) }\end{array}$ & USA & $491 / 1119$ & $30-49$ & $\mathrm{~F}$ & MI & $\begin{array}{l}\text { None } \\
1-4 \text { cups/day } \\
5-9 \text { cups/day } \\
\geq 10 \text { cups/day } \\
\text { ( } \geq 5 \text { cups/day) }\end{array}$ & $\begin{array}{l}1.00 \\
1.0(0.7-1.3) \\
1.4(1.0-2.1) \\
2.0(1.2-3.5) \\
1.7(1.2-2.4)\end{array}$ & $\begin{array}{l}\text { Age, smoking habit, } \\
\text { hypertension, diabetes, } \\
\text { hypercholesterolemia, } \\
\text { BMI, oral contraceptive } \\
\text { use }\end{array}$ \\
\hline $\begin{array}{l}\text { Rosenberg et al. } \\
1988 \text { [9] }\end{array}$ & USA & $1867 / 1158$ & $25-54$ & M & MI & $\begin{array}{l}\text { None } \\
1-2 \text { cups/day } \\
3-4 \text { cups/day } \\
5-9 \text { cups/day } \\
\geq 10 \text { cups/day } \\
\text { ( } \geq 5 \text { cups/day) }\end{array}$ & $\begin{array}{l}1.00 \\
1.4(1.0-1.9) \\
1.6(1.2-2.2) \\
1.8(1.3-2.5) \\
2.9(2.9-4.3) \\
2.2(1.6-3.0)\end{array}$ & $\begin{array}{l}\text { Age, smoking habit, } \\
\text { hypertension, diabetes, } \\
\text { hypercholesterolemia, } \\
\text { BMI, physical activity, } \\
\text { alcohol, family history } \\
\text { of MI, education }\end{array}$ \\
\hline $\begin{array}{l}\text { Kalandidi } \\
\quad \text { et al. } 1992 \text { [10] }\end{array}$ & Greece & $329 / 570$ & $<40 ;>80^{\mathrm{a}}$ & $M / F$ & $\mathrm{CHD}$ & $\begin{array}{l}<1 \text { cups/day } \\
\geq 1-2 \text { cups/day } \\
\geq 3 \text { cups/day }\end{array}$ & $\begin{array}{l}1.00 \\
1.17(0.75-1.82) \\
2.07(1.18-3.61)\end{array}$ & $\begin{array}{l}\text { Age, gender, smoking } \\
\text { habit, hypertension, } \\
\text { diabetes, } \\
\text { hypercholesterolemia, } \\
\text { physical activity, alcohol }\end{array}$ \\
\hline Brown et al. 1993 [23] & Scotland & $2074 / 7494$ & $40-59$ & $M / F$ & $\mathrm{CHD}$ & $\begin{array}{l}\text { None } \\
1-2 \text { cups/day } \\
3-4 \text { cups/day } \\
\geq 5 \text { cups/day }\end{array}$ & $\begin{array}{l}1.00 \\
0.83(0.72-0.97) \\
0.81(0.68-0.97) \\
0.81(0.67-0.99)\end{array}$ & $\begin{array}{l}\text { Age, cholesterol, } \\
\text { diastolic blood pressure, } \\
\text { smoking habit, BMI, } \\
\text { total energy, alcohol }\end{array}$ \\
\hline D’Avanzo et al. 1993 [11] & Italy & $801 / 792$ & $24-74$ & M & MI & $\begin{array}{l}\text { Non-use } \\
1 \text { cup/day } \\
2 \text { cups/day } \\
3 \text { cups/day } \\
4 \text { cups/day } \\
\geq 5 \text { cups/day }\end{array}$ & $\begin{array}{l}1.00 \\
0.8(0.5-1.2) \\
1.3(0.9-2.0) \\
1.8(1.1-2.7) \\
2.5(1.5 .4 .1) \\
2.6(1.6-4.2)\end{array}$ & $\begin{array}{l}\text { Age, smoking habit, } \\
\text { hypertension, diabetes, } \\
\text { hypercholesterolemia, } \\
\text { BMI, alcohol, family } \\
\text { history of } \mathrm{MI} \text {, education }\end{array}$ \\
\hline Palmer et al. 1995 [5] & USA & $858 / 858$ & $45-69$ & $\mathrm{~F}$ & MI & $\begin{array}{l}\leq 1 \mathrm{cup} / \mathrm{day} \\
1-2 \mathrm{cups} / \mathrm{day} \\
3-4 \mathrm{cups} / \mathrm{day} \\
5-6 \mathrm{cups} / \mathrm{day} \\
7-9 \mathrm{cups} / \mathrm{day} \\
\geq 10 \mathrm{cups} / \mathrm{day} \\
(\geq 5 \mathrm{cups} / \mathrm{day})\end{array}$ & $\begin{array}{l}1.00 \\
0.9(0.6-1.3) \\
0.9(0.6-1.4) \\
1.4(0.8-2.5) \\
2.1(0.9-4.9) \\
2.5(1.0-6.5) \\
1.7(1.0-1.7)\end{array}$ & $\begin{array}{l}\text { Age, smoking habit, } \\
\text { hypertension, diabetes, } \\
\text { family history of MI, } \\
\text { BMI, education, alcohol, } \\
\text { physical activity }\end{array}$ \\
\hline
\end{tabular}




\begin{tabular}{|c|c|c|c|c|c|c|c|c|}
\hline Rabajoli et al. 1997 [12] & Italy & $88 / 136^{b}$ & $35-70$ & M & MI & $\begin{array}{l}<1 \text { cup/day } \\
1-2 \text { cups/day } \\
2-3 \text { cups/day } \\
3-4 \text { cups/day } \\
>4 \text { cups/day }\end{array}$ & $\begin{array}{l}1.00 \\
1.1(0.48-2.23) \\
2.8(0.66-3.84) \\
4.0(0.70-7.96) \\
5.7(2.0-26.9)\end{array}$ & Age, BMI, hypertension \\
\hline Sesso et al. 1999 [22] & USA & $336 / 337$ & $<76^{a}$ & $M / F$ & MI & $\begin{array}{l}\leq 1 \text { cup } / \text { week } \\
>1-3 \mathrm{cups} / \text { day } \\
\geq 4 \text { cups/day }\end{array}$ & $\begin{array}{l}1.00 \\
0.91(0.58-1.45) \\
0.84(0.49-1.42)\end{array}$ & $\begin{array}{l}\text { Age, gender, smoking habit, } \\
\text { hypertension, family history of } \\
\text { MI, diabetes, BMI, physical activity, } \\
\text { total caloric intake, alcohol }\end{array}$ \\
\hline Tavani et al. 2001 [21] & Italy & $507 / 478$ & $25-79$ & $M / F$ & MI & $\begin{array}{l}\leq 1 \text { cup/day } \\
1-2 \text { cups/day } \\
>2-3 \text { cups/day } \\
>3-5 \text { cups/day } \\
\geq 6 \text { cups/day }\end{array}$ & $\begin{array}{l}1.00 \\
1.4(0.9-2.1) \\
1.1(0.7-1.7) \\
1.6(1.0-2.5) \\
1.9(1.1-3.3)\end{array}$ & $\begin{array}{l}\text { Age, gender, smoking } \\
\text { habit, hypertension, } \\
\text { diabetes, hypercholesterolemia, } \\
\text { BMI, history of MI, alcohol, } \\
\text { physical activity }\end{array}$ \\
\hline $\begin{array}{l}\text { Panagiotakos } \\
\text { et al. } 2003 \text { [42] }\end{array}$ & Greece & $848 / 1078$ & $56-65$ & $M / F$ & ACS & $\begin{array}{l}\text { None } \\
1-2 \text { cups/day } \\
2-4 \text { cups/day } \\
>4 \text { cups/day }\end{array}$ & $\begin{array}{l}1.00 \\
0.69(0.5-0.86) \\
1.56(1.1-2.34) \\
3.1(1.82-5.26)\end{array}$ & $\begin{array}{l}\text { Age, gender, smoking habit, } \\
\text { hypertension, diabetes, } \\
\text { hypercholesterolemia, BMI, } \\
\text { alcohol, family history of } \mathrm{MI} \text {, } \\
\text { education }\end{array}$ \\
\hline Tavani et al. 2004 [32] & Italy & $558 / 1044$ & $18-79$ & $\mathrm{~F}$ & MI & $\begin{array}{l}<1 \text { cup/day } \\
1-3 \text { cups/day } \\
>3 \text { cups/day }\end{array}$ & $\begin{array}{l}1.00 \\
1.1(0.8-1.6) \\
1.4(0.9-2.1)\end{array}$ & $\begin{array}{l}\text { Age, smoking habit, hypertension, } \\
\text { diabetes, dyslipidemia, BMI, study, } \\
\text { education, alcohol, physical } \\
\text { activity, family history of MI }\end{array}$ \\
\hline $\begin{array}{l}\text { Azevedo and } \\
\text { Barros } 2006 \text { [26] }\end{array}$ & Portugal & $290 / 364^{c}$ & $>40^{a}$ & $M / F$ & MI & $\begin{array}{l}\text { None } \\
\leq 2 \text { cups/day } \\
>2-4 \text { cups/day } \\
>4 \text { cups/day }\end{array}$ & $\begin{array}{l}1.00 \\
2.04(0.28-15.03) \\
2.44(0.31-19.03) \\
1.72(0.20-14.46)\end{array}$ & $\begin{array}{l}\text { Age, education, smoking habit, } \\
\text { waist-to-hip ratio }\end{array}$ \\
\hline
\end{tabular}


Table 2 Characteristics of cohort prospective studies included in the meta-analysis

\begin{tabular}{|c|c|c|c|c|c|c|c|c|c|}
\hline Source, year & Country & $\begin{array}{l}\text { Total } \\
\mathrm{N} / \text { no. of } \\
\text { cases }\end{array}$ & $\begin{array}{l}\text { Mean Follow-up } \\
\text { (years) }\end{array}$ & $\begin{array}{l}\text { Age at } \\
\text { baseline } \\
\text { (years) }\end{array}$ & Gender & Outcome & $\begin{array}{l}\text { Coffee } \\
\text { consumption }\end{array}$ & $\begin{array}{l}\text { Adjusted } \\
\text { RR }(95 \% \mathrm{Cl})\end{array}$ & Adjustment \\
\hline $\begin{array}{l}\text { LaCroix } \\
\text { et al. } 1986 \text { [20] }\end{array}$ & USA & $1040 / 47$ & $19-35$ & $19-49$ & $M$ & $\mathrm{CHD}$ & $\begin{array}{l}\text { None } \\
1-2 \text { cups/day } \\
3-4 \mathrm{cups} / \text { day } \\
\geq 5 \text { cups/day }\end{array}$ & $\begin{array}{l}1.00 \\
1.19(0.93-1.51) \\
1.49(0.85-2.63) \\
1.77(0.79-3.98)\end{array}$ & $\begin{array}{l}\text { Age, cholesterol, } \\
\text { smoking habit }\end{array}$ \\
\hline $\begin{array}{l}\text { Klatsky } \\
\text { et al. } 1990 \text { [43] }\end{array}$ & USA & $101774 / 1914$ & 5 & $59^{a}$ & $M / F$ & MI & $\begin{array}{l}\text { None } \\
<1 \text { cup/day } \\
1-3 \text { cups/day } \\
\geq 4 \text { cups/day }\end{array}$ & $\begin{array}{l}1.00 \\
0.78(0.56-1.07) \\
1.16(0.93-1.45) \\
1.42(1.11-1.81)\end{array}$ & $\begin{array}{l}\text { Age, smoking } \\
\text { habit, alcohol, } \\
\text { education, } \\
\text { baseline diseases }\end{array}$ \\
\hline $\begin{array}{l}\text { Lindsted } \\
\text { et al. } 1992 \text { [14] }\end{array}$ & USA & $9484 / 647$ & 15 & $50-99$ & $M$ & CHD & $\begin{array}{l}<1 \mathrm{cup} / \text { day } \\
1-2 \mathrm{cups} / \text { day } \\
\geq 3 \mathrm{cups} / \text { day }\end{array}$ & $\begin{array}{l}1.00 \\
1.33(1.15-1.54) \\
1.35(1.08-1.68)\end{array}$ & $\begin{array}{l}\text { Age, BMI, } \\
\text { hypertension, } \\
\text { smoking habit, } \\
\text { exercise, other } \\
\text { diseases, } \\
\text { dietary pattern }\end{array}$ \\
\hline $\begin{array}{l}\text { Klatsky } \\
\text { et al. } 1993 \text { [24] }\end{array}$ & USA & $128934 / 433$ & $3-10$ & $\begin{array}{l}<50 ; \geq 60 \text { (not } \\
\text { otherwise } \\
\text { indicated) }\end{array}$ & $\mathrm{M} / \mathrm{F}$ & MI & $\begin{array}{l}\text { None } \\
<1 \text { cup/day } \\
1-3 \text { cups/day } \\
\geq 4 \text { cups } / \text { day }\end{array}$ & $\begin{array}{l}1.00 \\
0.99(0.67-1.46) \\
1.17(0.87-1.57) \\
1.35(0.97-1.89)\end{array}$ & $\begin{array}{l}\text { Age, gender, BMI, } \\
\text { smoking habit, } \\
\text { alcohol, education }\end{array}$ \\
\hline $\begin{array}{l}\text { Klag } \\
\text { et al. } 1994 \text { [4] }\end{array}$ & USA & $933 / 95$ & $28-44$ & $26.3^{a}$ & M & $\mathrm{CHD}$ & $\begin{array}{l}\text { None } \\
1-2 \text { cups/day } \\
3-4 \mathrm{cups} / \text { day } \\
>5 \mathrm{cups} / \text { day }\end{array}$ & $\begin{array}{l}1.00 \\
1.70(0.78-3.68) \\
3.02(1.37-6.65) \\
2.94(1.27-6.81)\end{array}$ & $\begin{array}{l}\text { Age, cholesterol, } \\
\text { smoking habit, } \\
\text { hypertension, } \\
\text { diabetes, BMI }\end{array}$ \\
\hline $\begin{array}{l}\text { Gyntelberg } \\
\text { et al. } 1995 \text { [17] }\end{array}$ & Denmark & $2975 / 184$ & 6 & $53-74$ & $M$ & $\mathrm{CHD}$ & $\begin{array}{l}1-4 \text { cups/day } \\
5-8 \mathrm{cups} / \text { day } \\
\geq 9 \mathrm{cups} / \text { day }\end{array}$ & $\begin{array}{l}1.00 \\
1.0(0.7-1.4) \\
0.6(0.3-1.0)\end{array}$ & $\begin{array}{l}\text { Age, smoking } \\
\text { habit, alcohol, } \\
\text { hypertension, } \\
\text { triglycerides }\end{array}$ \\
\hline $\begin{array}{l}\text { Hart and } \\
\text { Smith, } 1997 \text { [25] }\end{array}$ & Scotland & $2686 / 282$ & 21 & $35-64$ & $M$ & CHD & $\begin{array}{l}\text { None } \\
0.5-1 \text { cups/day } \\
1.5-2 \text { cups/day } \\
2.5-4 \text { cups/day } \\
>4.5 \text { cups/day }\end{array}$ & $\begin{array}{l}1.00 \\
1.20(0.87-1.64) \\
1.17(0.83-1.65) \\
1.16(0.81-1.66) \\
1.49(0.89-2.47)\end{array}$ & $\begin{array}{l}\text { Age, diastolic } \\
\text { blood pressure, } \\
\text { cholesterol, } \\
\text { smoking habit, } \\
\text { BMI, social class, } \\
\text { education }\end{array}$ \\
\hline
\end{tabular}




\begin{tabular}{|c|c|c|c|c|c|c|c|c|c|}
\hline $\begin{array}{l}\text { Lopez-Garcia } \\
\text { et al. } 2006 \text { [27] } \\
\text { (final data of Willett } \\
\text { et al. } 1996[18] \text { ) }\end{array}$ & USA & $84488 / 2254$ & 20 & $34-59$ & $F$ & $\mathrm{CHD}$ & $\begin{array}{l}<1 \text { cup/month } \\
1 / \text { month-4 } \\
\text { cups / week } \\
5-7 \text { cups/week } \\
2-3 \text { cups/day } \\
4-5 \mathrm{cups} / \text { day } \\
\geq 6 \text { cups/day }\end{array}$ & $\begin{array}{l}1.00 \\
0.97(0.83-1.14) \\
1.02(0.90-1.17) \\
0.84(0.74-0.97) \\
0.99(0.83-1.17) \\
0.87(0.68-1.11)\end{array}$ & $\begin{array}{l}\text { Age, smoking } \\
\text { habit, BMI, } \\
\text { diabetes, } \\
\text { hypertension, } \\
\text { hypercholesterolemia, } \\
\text { family history } \\
\text { of MI, alcohol, } \\
\text { physical activity, } \\
\text { menopausal status, } \\
\text { hormone therapy, } \\
\text { aspirin use }\end{array}$ \\
\hline $\begin{array}{l}\text { Lopez-Garcia } \\
\text { et al. } 2006 \text { [27] } \\
\text { (final data of } \\
\text { Grobbee et al. [16]) }\end{array}$ & USA & $44005 / 2173$ & 14 & $40-84$ & M & $\mathrm{CHD}$ & $\begin{array}{l}<1 \text { cup/month } \\
1 / \text { month-4 } \\
\quad \text { cups / week } \\
5-7 \text { cups/week } \\
2-3 \text { cups/day } \\
4-5 \mathrm{cups} / \text { day } \\
\geq 6 \mathrm{cups} / \text { day }\end{array}$ & $\begin{array}{l}1.00 \\
1.04(0.91-1.17) \\
1.02(0.91-1.15) \\
0.97(0.86-1.11) \\
1.07(0.88-1.31) \\
0.72(0.49-1.07)\end{array}$ & $\begin{array}{l}\text { Age, smoking } \\
\text { habit, BMI, diabetes, } \\
\text { hypertension, } \\
\text { hypercholesterolemia, } \\
\text { family history of MI, } \\
\text { alcohol, physical } \\
\text { activity, aspirin use }\end{array}$ \\
\hline $\begin{array}{l}\text { Andersen } \\
\text { et al. } 2006 \text { [28] }\end{array}$ & U.S.A & $27312 / 1411$ & 15 & $55-69$ & $F$ & $\mathrm{CHD}$ & $\begin{array}{l}\text { None } \\
<1 \text { cups/day } \\
1-3 \text { cups/day } \\
4-5 \text { cups/day } \\
\geq 6 \mathrm{cups} / \text { day }\end{array}$ & $\begin{array}{l}1.00 \\
0.85(0.68-1.06) \\
0.76(0.64-0.91) \\
0.81(0.66-0.99) \\
0.87(0.69-1.04)\end{array}$ & $\begin{array}{l}\text { Age, smoking habit, } \\
\text { BMI, waist-hip ratio, } \\
\text { education, physical } \\
\text { activity, alcohol, } \\
\text { use of estrogens, } \\
\text { energy intake, } \\
\text { multivitamin } \\
\text { supplements, dietary } \\
\text { pattern }\end{array}$ \\
\hline
\end{tabular}


(if present) would explain the observed associations, we calculated fail-safe numbers that indicated the number of studies with null results that would need to be added to the meta-analysis in order to reduce the overall level of significance of the observed result to that of non-significance [66].

\section{Results}

\section{Case-control studies}

Characteristics of the case-control studies included in the meta-analysis are described in Table 1. The vast majority of these studies had myocardial infarction as the primary outcome. Fig. 1 shows the results of case-control studies for different levels of coffee consumption, compared with the lowest level of coffee consumption. First, we compared the highest category of coffee consumption with the reference category. The summary OR from a random-effect model showed that individuals who consumed a high amount of coffee per day had an increased risk of developing CHD (OR 1.83; 95\% Cl 1.49-2.24; $P<0.0001$ ) (Fig. 1a). However, the $P$ value for heterogeneity was 0.02 . Studies by Sesso et al. [22] and by Brown et al. [23] contributed substantially to the heterogeneity in this result. Nevertheless, exclusion of these studies from the analysis did not change the significant association found between the highest category of coffee consumption and CHD (OR 2.03; 95\% Cl 1.77-2.33; $P<0.0001$ ); $P$ for heterogeneity $=0.46$.

We subsequently compared the second highest category of coffee consumption versus the reference category, showing that the overall effect of a moderately high consumption of coffee remained significantly associated with the occurrence of CHD (OR 1.33; 95\% Cl 1.04-1.71; $P<0.0001) ; P$ for heterogeneity $<0.0001$ (Fig. 1b). Exclusion of the study by Brown et al. [23], which accounted for the significant heterogeneity demonstrated in this model, did not influence the overall result (OR 1.42; 95\% Cl 1.18-1.71; $P=0.002$ ); $P$ for heterogeneity $=0.06$.

Finally, as the third highest category of coffee consumption ( $\leq 2$ cups per day) was analysed, the significant association between coffee consumption and CHD found for the other categories was not present (OR 1.03; 95\% Cl 0.87-1.21; $P=0.76$ ) (Fig. 1c). The $P$ for heterogeneity was significant $(P=0.01)$; however, after exclusion of the study by Brown et al. [23], which accounted for significant heterogeneity in the model, the results did not change substantially (OR $1.07 ; 95 \% \mathrm{Cl}$ $0.89-1.29 ; P=0.46$ ); $P$ for heterogeneity $=0.06$.

\section{Cohort studies}

Characteristics of the cohort studies included in the meta-analysis are described in Table 2. In contrast to case-control studies, many of the cohort prospective studies reported CHD as the primary outcome and involved male subjects.

In 10 cohort studies, including a total of 403,631 participants followed for between 3 and 44 years, the cumulative RR for all cohort studies was 1.16 (95\% Cl $0.95-1.41 ; P=0.14)$ for the highest category of coffee consumption (Fig. 2a), thus not showing an association between high coffee consumption and CHD. However, the analysis showed a significant heterogeneity across all studies $(P=0.0008)$, mainly due to the studies by Klatsky et al. [35] and Lindsted et al. [14], which accounted for the largest RRs. By excluding these studies from the analysis, the result was confirmed (RR $1.11 ; 95 \% \mathrm{Cl} 0.88-1.41 ; P=0.38) ; P$ for heterogeneity $=0.08$.

We analysed the second highest category of coffee consumption, representing a moderate daily intake of coffee, and the summary RR did not reveal a significant association between coffee consumption and CHD (RR 1.05; 95\% Cl 0.90-1.22; $P=0.57$ ) (Fig. 2b). The result was confirmed after exclusion from the analysis of the study by Klag et al. [4], which accounted for the heterogeneity in the model (RR 1.04; 95\% Cl 0.89-1.20; $P=0.4$ ).

Finally, we performed the analysis for the third highest category of coffee consumption ( $\leq 2$ cups per day), and found that the cumulative RR for all cohort studies was 1.04 (95\% Cl 0.90-1.19; $P=0.60$ ) (Fig. 2c). A significant heterogeneity across all studies was reported $(P=0.002)$; however, after eliminating from the model the study by Lindsted et al. [14], which accounted for significant heterogeneity, the result did not differ substantially (RR 0.99; 95\% Cl 0.87-1.12; $P=0.85$ ); $P$ for heterogeneity $=0.34$.

\section{Characteristics of the study population}

Similar associations for coffee consumption categories among US compared with European casecontrol and cohort studies were seen (Table 3 ). In contrast, cohort prospective studies published before the median year of publication of all of the studies included, report an association with CHD while those published after the median year. Finally, no significant differences, according to fatal and non-fatal events as well as to the number of years of follow-up within cohort studies, were observed. 


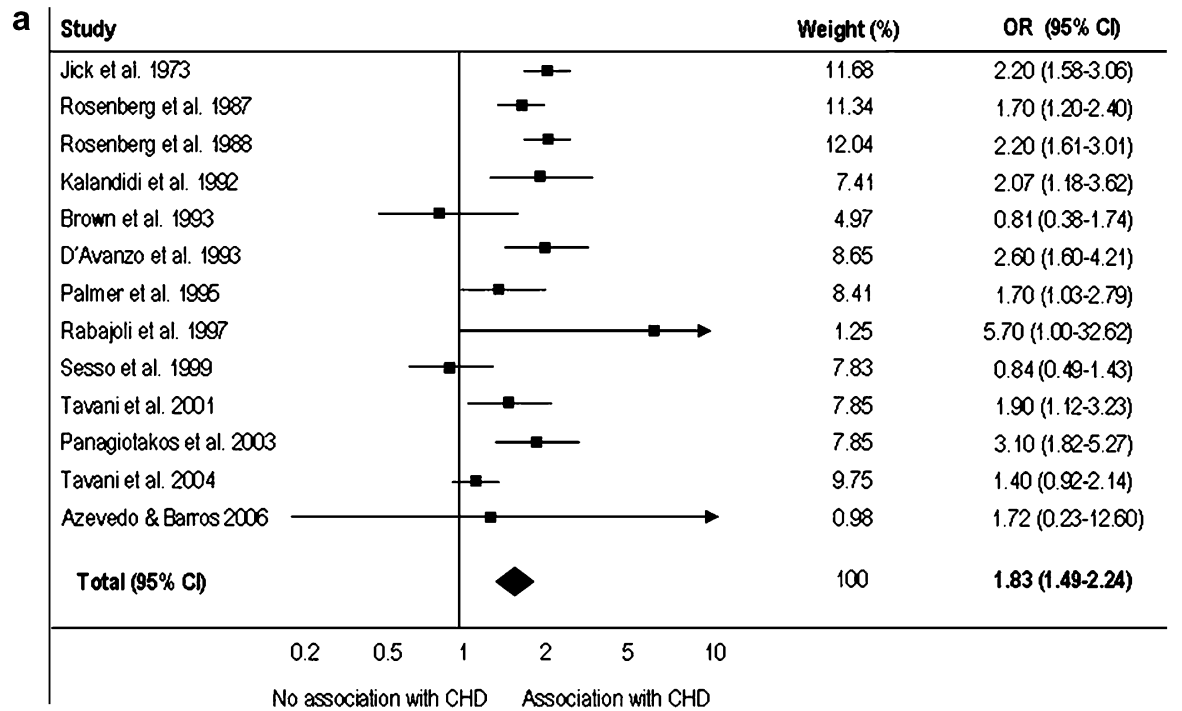

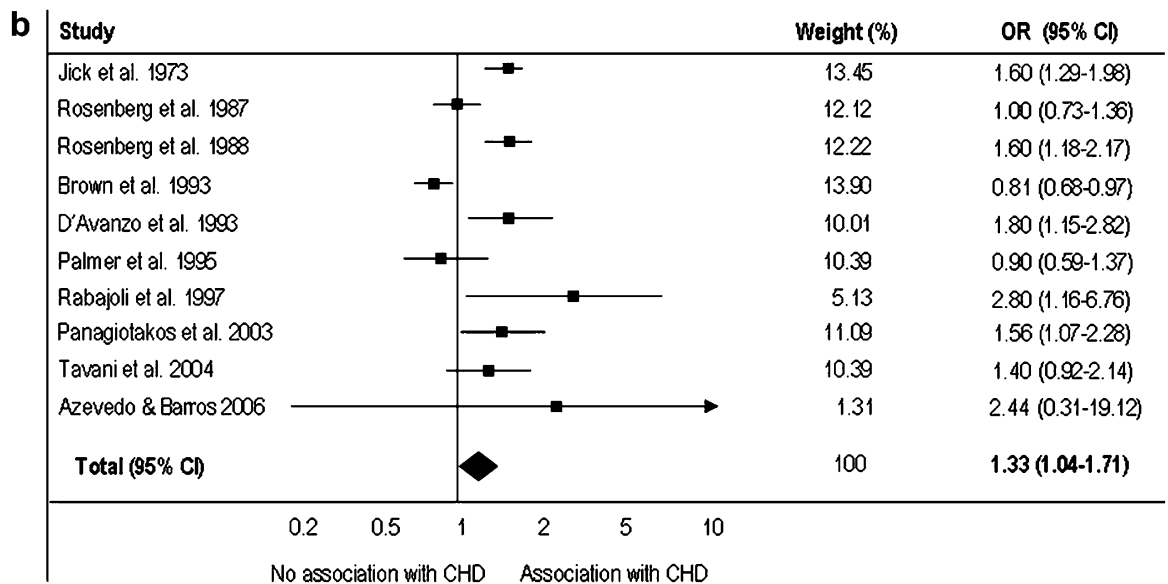

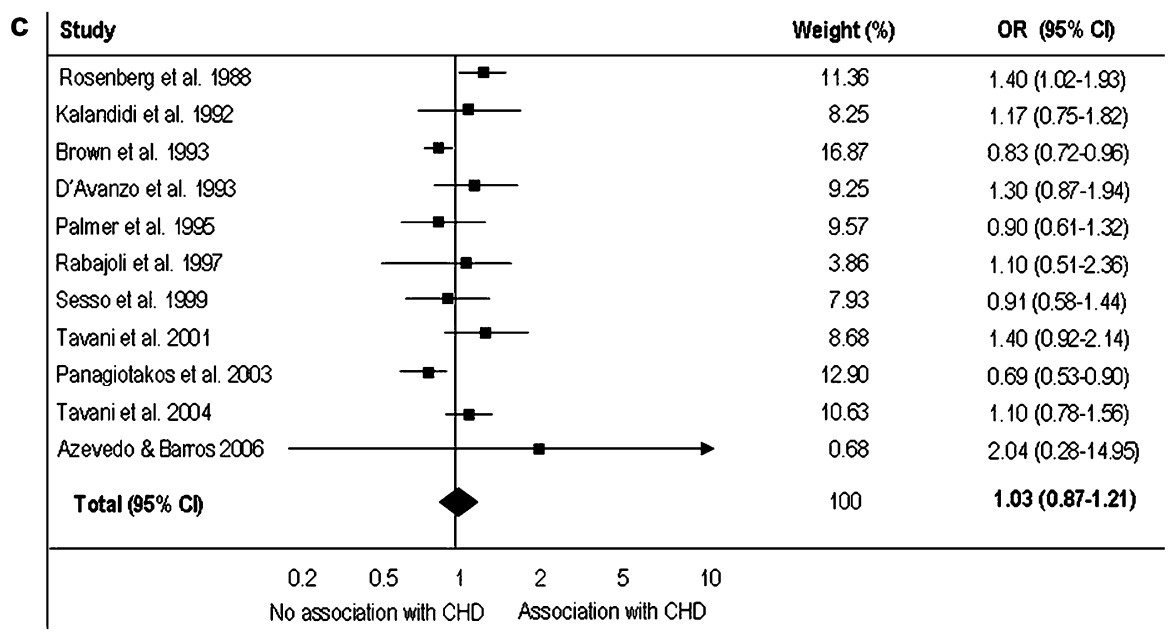

Figure 1 Odds ratios for the association between coffee consumption and coronary heart disease among casecontrol studies. (a) Highest category of coffee consumption ( $>4$ cups/day) vs. reference category (none or $<1$ cup/day). (b) Second highest category of coffee consumption (3-4 cups/day) vs. reference category (none or $<1$ cup/day). (c) Third highest category of coffee consumption ( $\leq 2$ cups/day) vs. reference category (none or $<1$ cup/day). 


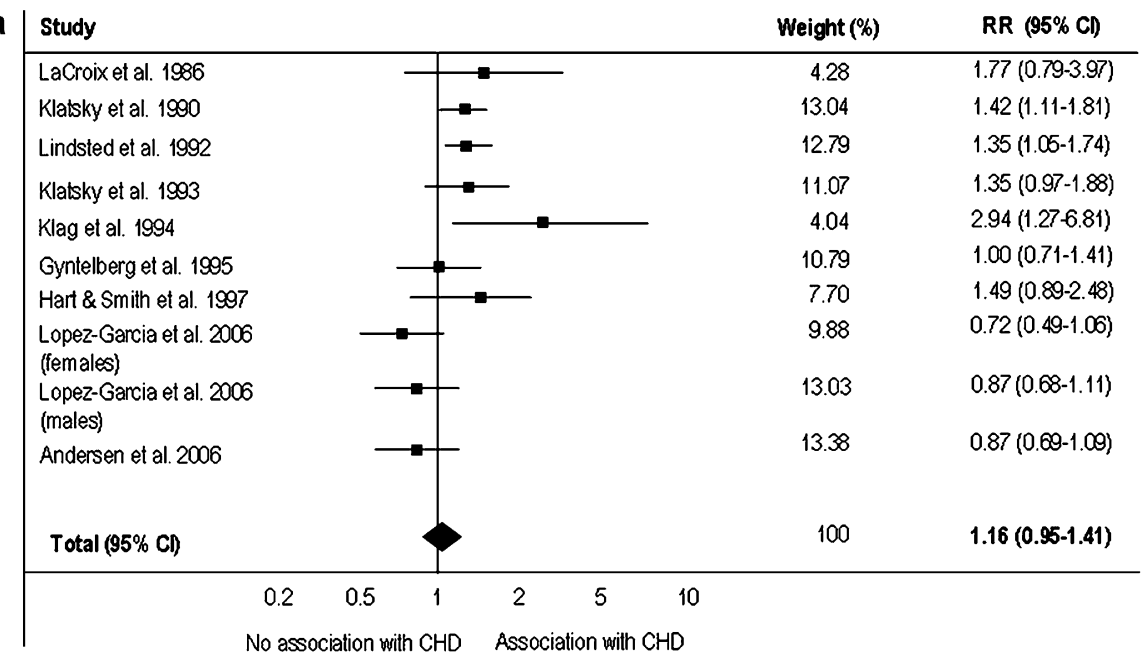

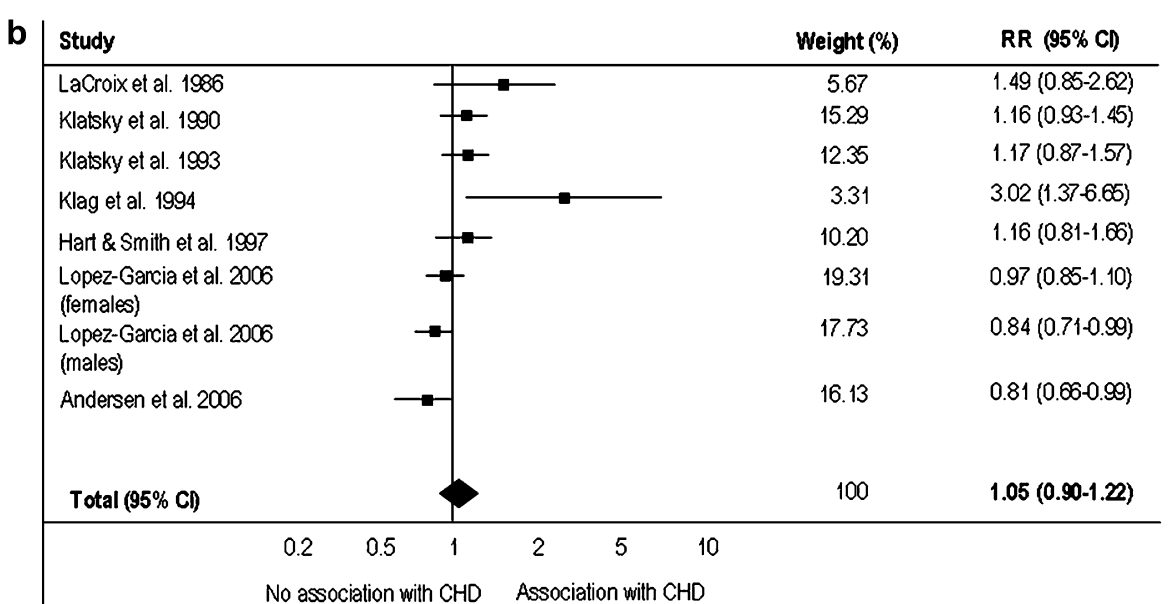

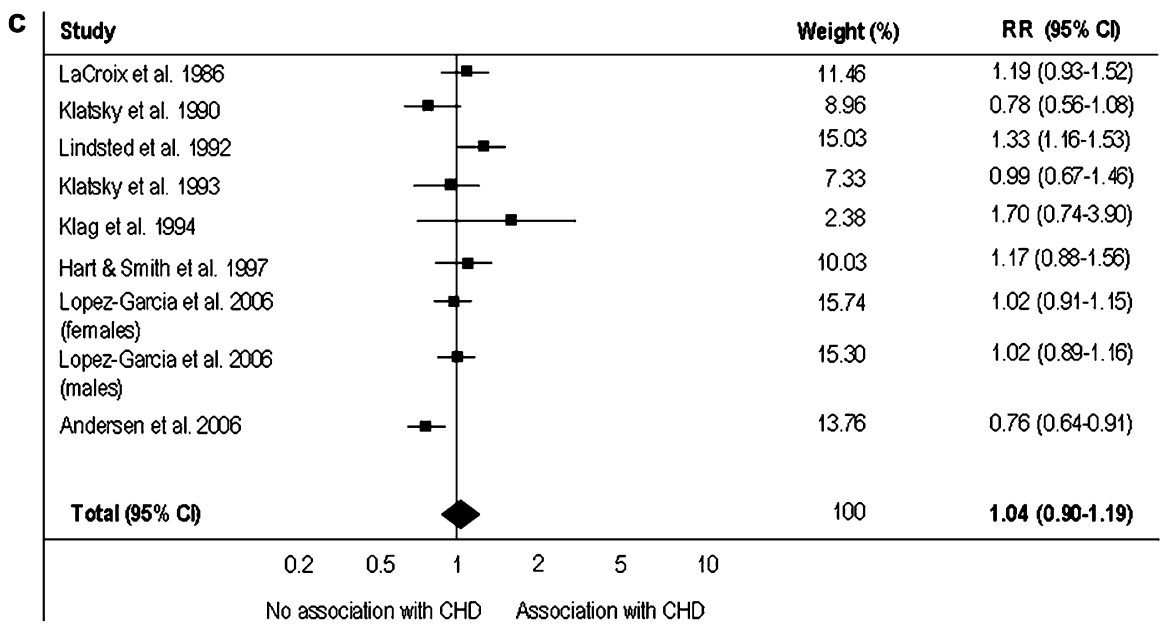

Figure 2 Relative risks for the association between coffee consumption and coronary heart disease among cohort prospective studies. (a) Highest category of coffee consumption ( $>4$ cups/day) vs. reference category (none or $<1$ cup/ day). (b) Second highest category of coffee consumption (3-4 cups/day) vs. reference category (none or $<1$ cup/ day). (c) Third highest category of coffee consumption ( $\leq 2$ cups/day) vs. reference category (none or $<1$ cup/day). 


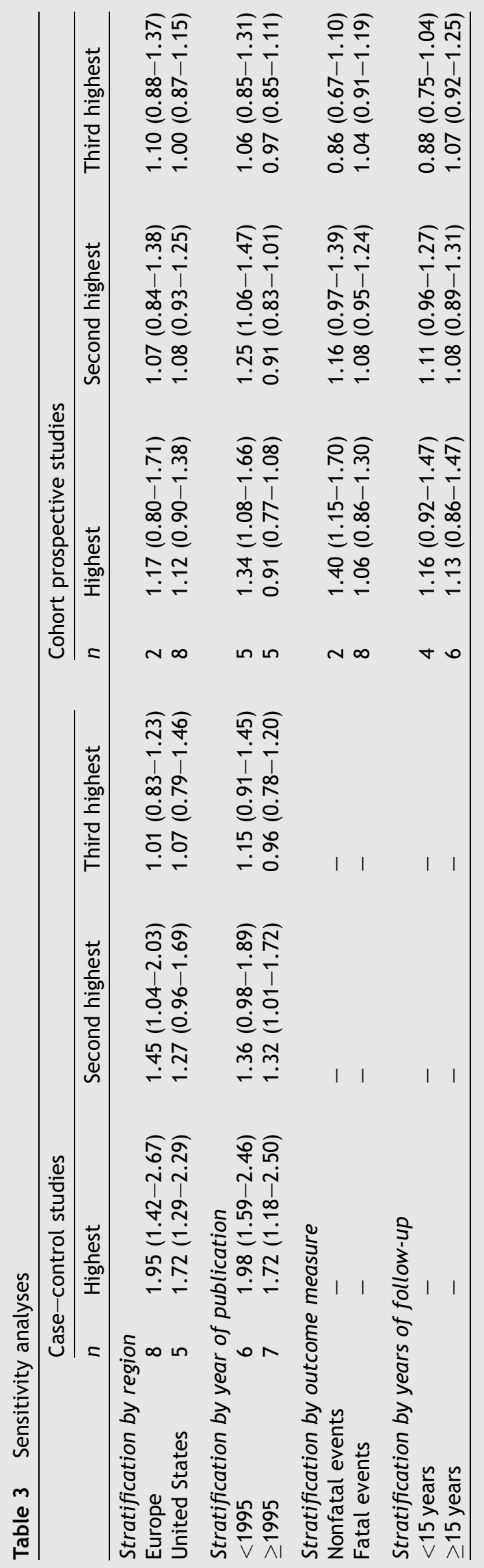

\section{Assessment of publication bias}

We explored the potential for publication bias using funnel plots of effect size versus standard error. The plots for all the categories of coffee consumption among case-control studies were broadly symmetrical, consistent with the conclusion that there was no major publication bias, whereas slightly asymmetrical plots for cohort studies were observed. Thus, we calculated the number of studies with null results that would need to be added to the meta-analysis to reduce the overall observed associations to a level of nonsignificance, i.e. the fail-safe number. This was 228 for the highest level of coffee consumption among case-control studies, and nine for the highest category of consumption within cohort studies. Hence, the number was robust for casecontrol studies only, according to the commonly used criterion that an analysis requires a fail-safe number greater than $5 n+10$ (where $n$ is the original number of studies in the analysis). However, by performing sensitivity analyses among cohort studies, it could be observed that smaller cohort studies published before 1995 are the principle determinants of the low fail-safe number reported.

\section{Decaffeinated coffee}

Decaffeinated coffee was examined separately in four case-control studies $[5,8,9,22]$ and three cohort studies $[27,28]$ included in the meta-analysis. In addition, a paper by La Vecchia et al. [44] eliminated from the previous analysis since it investigated only decaffeinated coffee, was introduced into the present model at this point. Within case-control studies consumption of decaffeinated coffee, in terms of both the highest and lowest categories ( $>4$ cups per day and $\leq 2$ cups per day, respectively), was not significantly associated with an increased risk of CHD (OR 1.87; $95 \% \mathrm{Cl}$ $0.96-3.46 ; P=0.07$ and $\mathrm{OR} 1.05 ; 95 \% \mathrm{Cl} 0.85-$ $1.29 ; P=0.7$, respectively); heterogeneity across the studies was also not significant. Similarly, no significant association between decaffeinated coffee consumption and CHD was reported for cohort studies, at both categories of consumption (RR 1.10; $95 \% \mathrm{Cl} 0.90-1.34 ; P=0.33$ and $\mathrm{RR} 1.00$; 95\% $\mathrm{Cl}$ 0.93-1.07; $P=0.90$, respectively).

\section{Discussion}

The present meta-analysis, performed in 13 casecontrol and 10 cohort studies, with an overall 
incorporated population of more than 430,000 subjects and 18,000 CHD cases, shows no clear indication that coffee consumption determines an increased risk of CHD. An association among the highest categories (mainly $>2$ cups per day) of coffee drinking and CHD within case-control studies has been reported. However, this result was not confirmed among cohort prospective studies with a follow-up period ranging from 3 to 44 years. Nevertheless, it should be noted that among cohort prospective studies, publication bias cannot be excluded because of the low fail-safe number assessed. Finally, decaffeinated coffee, analysed in four case-control and three cohort studies, showed no significant association between both the highest and the third highest categories of coffee consumption and an increased risk of CHD, with respect to the reference category.

During the past three decades, the relationship between coffee drinking and the occurrence of CHD has been assessed in numerous studies, with conflicting results [1-28]. Some studies reported a positive association between coffee intake and CHD [1-14], whereas others did not [15-28], possibly suggesting that the increase in the susceptibility of coronary disease found in previous studies was the result of either an unhealthy lifestyle pattern associated with coffee consumption, or of some detrimental acute effects of coffee intake on CHD-related risk factors. Coffee has been widely investigated for its effects on the cardiovascular system, and the biological plausibility of an increased risk of CHD with a high intake of coffee has been identified through its influence on blood pressure, serum lipids, inflammatory markers, and homocysteine-some of the most important risk factors for CHD. Administration of coffee has been demonstrated to significantly raise blood pressure in men [67], but not in women [68]. Moreover, coffee has been reported to raise inflammatory markers and lipid levels, although the effect on lipids is probably influenced by the brewing method, being higher for the boiled compared to the filtered method $[36,69]$. Finally, some studies during recent years have reported a significant influence of coffee beverages on plasma levels of homocysteine [70]. On the other hand, a protective effect of coffee consumption on the occurrence of diabetes has been suggested by a recent metaanalysis, which showed a significant inverse association between habitual coffee consumption and the risk of type 2 diabetes [71]. Similarly, coffee consumption has been found to be associated with a reduced risk of chronic liver disease [72].

Three comprehensive meta-analyses investigating coffee consumption in relation to CVD were published previously, reporting conflicting findings. In 1992, Myers et al. [29] reported that in 11 prospective cohort studies there was no evidence for an association between coffee consumption and the development of CHD; whereas in 1993, Greenland et al. [30] analysed eight case-control and 14 cohort studies, and observed that subjects drinking more than five cups per day had an increased risk of myocardial infarction if compared to non-drinkers, but only among case-control studies. On the other hand, another meta-analysis by Kawachi et al. [31] in 1994 assessing eight case-control and 15 cohort studies, concluded that a moderate-to-high coffee consumption was not significantly associated with an increased risk of coronary death or heart attack. The results of these meta-analyses are in keeping with our findings, since they all show no association between coffee consumption and the occurrence of CHD among cohort prospective studies, whereas a significant association is reported with regard to case-control studies. This divergence of results according to the different type of studies analysed could be ascribed to several factors: first, case-control studies suffer from recall bias, which may explain the positive association shown with the disease as individuals who developed CHD are more likely to over-report coffee intake than are healthy controls. In addition, case-control studies are not likely to include fatal events, whereas cohort studies usually do. This equates to a substantial difference in outcome measures between the two types of studies. Indeed, a recent analysis in the lowa Women's Study suggests that coffee may even be protective for fatal events, possibly contributing to differences between cohort and case-control studies [28]. Furthermore, caffeine has mainly adverse acute effects on the cardiovascular system, thus explaining the association with CHD found in case-control but not in follow-up studies. Alternatively, some of the discrepancies among studies on coffee and CHD could derive from the complex, heterogeneous, and controversial nature of coffee beverages. There are a wide variety of beverages containing caffeine, and the difference in terms of caffeine content between different types of coffees is extremely significant. In the present meta-analysis we used the estimation of coffee intake as cups consumed per day. This is the unit of measure generally reported in studies considering beverages like coffee and tea. However, there is no standard definition of cup size, and volumes may vary considerably among studies. Similarly, caffeine content is likely to be substantially different among the different kinds of coffee [73]. For instance, espresso coffee, 
commonly used in Italy, contains $35-45 \%$ less caffeine than percolated and boiled coffees, widely used in all other countries. Moreover, the effect of the different types of coffee on blood determinants related to CHD vary considerably: boiled coffee has been found extensively to be associated with an increase in serum cholesterol, whereas no association has been shown for coffee prepared by the filter process [36]. In our meta-analysis we did not find any significant differences when taking into account the regions of origin of the studies or the different types of coffees usually consumed across the different countries. In keeping with our results, a previous meta-analysis by Myers et al. showed no association between the method of coffee preparation and the occurrence of CHD [29]. Another possible explanation for the discrepancies in results could be related to the wide interindividual variability in coffee metabolism present among the population as a whole. Interestingly, it has been reported recently that a genetic variant in the cytochrome P450 1A2 gene, which accounts for approximately $95 \%$ of caffeine metabolism, plays an important role in the association between coffee consumption and the occurrence of myocardial infarction [74]. Moreover, it has been shown that subjects carrying this genetic variant had slow caffeine metabolism, and consequently an increased risk for CHD. This finding suggests an environmental-genetic interaction for coffee consumption, which may further explain the conflicting results of studies investigating coffee intake in relation to CHD. Publication bias is present within cohort prospective studies and is a main limitation in the present meta-analysis, especially considering the low fail-safe numbers obtained. On the other hand, sensitivity analyses performed according to the main characteristics of the studies demonstrated a significant influence of smaller and older studies in determining such bias.

In conclusion, the present meta-analysis indicates that, in general, coffee consumption is not associated with an increased risk of CHD, whereas a significant association of CHD with high consumption of coffee is reported among case-control studies. Furthermore, decaffeinated coffee seems not to be associated with an increased risk of CHD, despite the fact that only a few, limited studies are available where decaffeinated coffee is evaluated separately. The influence of coffee on the health of the people is considered to be of great significance since coffee is one of the most commonly consumed beverages in Western countries. Hence, these results appear of extreme importance in clarifying the relationship between coffee consumption and CHD.

\section{Acknowledgments}

This research was submitted to a Call for Research on Coffee and Cardiovascular Diseases and selected for a grant by SINU, (Italian Society of Human Nutrition).

\section{References}

[1] Report from the Boston Collaborative Drug Surveillance Program. Coffee drinking and acute myocardial infarction. Lancet 1972;ii:1278-81.

[2] Jick H, Miettinen OS, Neff RK, Shapiro S, Heinonen OP, Slone D. Coffee and myocardial infarction. N Engl J Med 1973;289:63-7.

[3] LeGrady D, Dyer AR, Shekelle RB, Stamler J, Liu K, Paul O, et al. Coffee consumption and mortality in the Chicago Western Electric Company Study. Am J Epidemiol 1987; 126:803-12.

[4] Klag MJ, Mead LA, LaCroix AZ, Wang NY, Coresh J, Liang KY, et al. Coffee intake and coronary heart disease. Ann Epidemiol 1994;4:425-33.

[5] Palmer JR, Rosenberg L, Rao RS, Shapiro S. Coffee consumption and myocardial infarction in women. Am J Epidemiol 1995;141:724-31.

[6] Paul O, Lepper MH, Phelan WH, Dupertuis GW, MacMillan A, McKean $\mathrm{H}$, et al. A longitudinal study of coronary heart disease. Circulation 1963;28:20-31.

[7] Rosenberg L, Slone D, Shapiro S, Kaufman DW, Stolley PD, Miettinen OS. Coffee drinking and myocardial infarction in young women. Am J Epidemiol 1980;111:675-81.

[8] Rosenberg L, Werler MM, Kaufman DW, Shapiro S. Coffee drinking and myocardial infarction in young women: an update. Am J Epidemiol 1987;126:147-9.

[9] Rosenberg L, Palmer JR, Kelly JP, Kaufman DW, Shapiro S. Coffee drinking and nonfatal myocardial infarction in men under 55 years of age. Am J Epidemiol 1988;128:570-8.

[10] Kalandidi A, Tzonou A, Toupadaki N, Lan S-J, Koutis C, Drogari P, et al. A case-control study of coronary heart disease in Athens, Greece. Int J Epidemiol 1992;21:1074-80.

[11] D’Avanzo B, La Vecchia C, Tognoni G, Franceschi S, Franzosi MG, Nobili A, et al. on behalf of GISSI-EFRIM. Coffee consumption and risk of acute myocardial infarction in Italian males. Ann Epidemiol 1993;3:595-600.

[12] Rabajoli F, Martone T, Arneodo D, Balzola F, Leo L, Vineis P. Internal migration, coffee drinking, and nonfatal myocardial infarction in Italy. Arch Environ Health 1997; 52:129-33.

[13] Martin JB, Annegers JF, Curb JD, Heyden S, Howson C, Lee SE, et al. Mortality patterns among hypertensives by reported level of caffeine consumption. Prev Med 1988; 17:310-20.

[14] Lindsted KD, Kuzma JW, Anderson JL. Coffee consumption and cause-specific mortality. J Clin Epidemiol 1992;45:733-42.

[15] Klatsky AL, Friedman GD, Siegelaub AB. Coffee drinking prior to acute myocardial infarction. Results from the Kaiser-Permanente Epidemiologic Study of Myocardial Infarction. JAMA 1973;226:540-3.

[16] Grobbee DE, Rimm EB, Glovannucci E, Colditz G, Stampfer M, Willett W. Coffee, caffeine, and cardiovascular disease in men. N Engl J Med 1990;323:1026-32.

[17] Gyntelberg F, Hein HO, Suadicani P, Sorensen H. Coffee consumption and risk of ischaemic heart disease-a settled issue? J Intern Med 1995;237:55-61. 
[18] Willett WC, Stampfer MJ, Manson JE, Colditz GA, Rosner BA, Speizer FE, et al. Coffee consumption and coronary heart disease in women. A ten-year follow-up. JAMA 1996;275:458-62.

[19] Dawber TR, Kannel WB, Gordon T. Coffee and cardiovascular disease-observations from the Framinghan Study. N Engl J Med 1974;291:871-4.

[20] LaCroix A, Mead LA, Liang KY, Thomas CB, Pearson TA. Coffee consumption and the incidence of coronary heart disease. N Engl J Med 1986;315:977-82.

[21] Tavani A, Bertuzzi M, Negri E, Sorbara L, La Vecchia C. Alcohol, smoking, coffee and risk of non-fatal acute myocardial infarction in Italy. Eur J Epidemiol 2001;17:1131-7.

[22] Sesso HD, Gaziano JM, Buring JE, Hennekens CH. Coffee and tea intake and the risk of myocardial infarction. Am J Epidemiol 1999;149:162-7.

[23] Brown CA, Bolton-Smith C, Woodward M, Tunstall-Pedoe H. Coffee and tea consumption and the prevalence of coronary heart disease in men and women: results from the Scottish Heart Health Study. J Epidemiol Community Health 1993;47:171-5.

[24] Klatsky AL, Armstrong MA, Friedman GD. Coffee, tea, and mortality. Ann Epidemiol 1993;3:375-81.

[25] Hart C, Smith GD. Coffee consumption and coronary heart disease mortality in Scottish men: a 21-year follow-up study. J Epidemiol Community Health 1997;51:461-2.

[26] Azevedo A, Barros H. Coffee and myocardial infarction: heterogeneity of an association in Portuguese men. Eur $\mathrm{J}$ Cardiovasc Prev Rehabil 2006;13:268-73.

[27] Lopez-Garcia E, van Dam RM, Willett WC, Rimm EB, Manson JAE, Stampfer E, et al. Coffee consumption and coronary heart disease in men and women. A prospective cohort study. Circulation 2006;113:2045-53.

[28] Andersen LF, Jacobs DR Jr, Carlsen MH, Blomhoff R. Consumption of coffee is associated with reduced risk of death attributed to inflammatory and cardiovascular diseases in the lowa Women's Health Study. Am J Clin Nutr 2006;83: 1039-46.

[29] Myers MG, Babinski A. Coffee and coronary heart disease. Arch Intern Med 1992;152:1767-72.

[30] Greenland S. A meta-analysis of coffee, myocardial infarction, and coronary death. Epidemiology 1993;4:366-74.

[31] Kawachi I, Colditz GA, Stone CB. Does coffee drinking increase the risk of coronary heart disease? Results from a meta-analysis. Br Heart J 1994;72:269-75.

[32] Tavani A, Bertuzzi M, Gallus S, Negri E, La Vecchia C. Risk factors for non-fatal acute myocardial infarction in Italian women. Prev Med 2004;39:128-34.

[33] Wilhelmsen L, Tibblin G, Elmfeldt D, Wedel H, Werko L. Coffee consumption and coronary heart disease in middle-aged Swedish men. Acta Med Scand 1977;201:547-52.

[34] Hennekens CH, Drolette ME, Jesse MJ, Davies JE, Hutchison GB. Coffee drinking and death due to coronary heart disease. N Engl J Med 1976;294:633-6.

[35] Gramenzi A, Gentile A, Fasoli M, Negri E, Parazzini F, La Vecchia C. Association between certain foods and risk of acute myocardial infarction in women. BMJ 1990;300: 771-3.

[36] Hammar N, Andersson T, Alfredsson L, Reuterwall C, Nilsson T, Hallqvist J, et al. Association of boiled and filtered coffee with incidence of first nonfatal myocardial infarction: the SHEEP and the VHEEP study. J Intern Med 2003;253:653-9.

[37] Tofler OB, Froy S, Hickey G, Burke V. Coffee and coronary heart disease. Heart Lung Circulation 2001;10:116-20.

[38] Rosenberg L, Slone D, Shapiro S, Kaufman DW, Miettinen OS. Case-control studies of the acute effects of coffee upon the risk of myocardial infarction: problems in the selection of a hospital control series. Am J Epidemiol 1981;113:646-52.

[39] de Vreede-Swagemakers JJM, Gorgels APM, Wejinberg MP, Dubois-Arbouw WI, Golombeck B, van Ree JW, et al. Risk indicators for out-of-hospital cardiac arrest in patients with coronary heart disease. J Clin Epidemiol 1999;52: 601-7.

[40] Weinmann S, Siscovick DS, Raghunathan TE, Arbogast P, Smith $\mathrm{H}$, Bovbjerg VE, et al. Caffeine intake in relation to the risk of primary cardiac arrest. Epidemiology 1997; 8:505-8.

[41] La Vecchia C, D’Avanzo B, Negri E, Franceschi S, Gentile A, Tavani A. Decaffeinated coffee and acute myocardial infarction. A case-control study in Italian women. Ann Epidemiol 1993;3:601-4.

[42] Panagiotakos DB, Pitsavos C, Chrysohoou C, Kokkinos P, Toutouzas P, Stefanadis C. The J-shaped effect of coffee consumption on the risk of developing acute coronary syndromes: the CARDIO2000 case-control study. J Nutr 2003; 133:3228-32.

[43] Klatsky AL, Friedman GD, Armstrong MA. Coffee use prior to myocardial infarction restudied: heavier intake may increase the risk. Am J Epidemiol 1990;132:479-88.

[44] Tverdal A, Stensvold I, Solvoll K, Foss OP, Lund-Larsen P, Bjartveit K. Coffee consumption and death from coronary heart disease in middle aged Norwegian men and women. BMJ 1990;300:566-9.

[45] Stensvold I, Tverdal A. The relationship of coffee consumption to various self-reported cardiovascular events in middle-aged Norwegian men and women. Scand J Soc Med 1995;23:103-9.

[46] Stensvold I, Tverdal A, Jacobsen BK. Cohort study of coffee intake and death from coronary heart disease. BMJ 1996; 312:544-5.

[47] Yano K, Rhoads GG, Kagan A. Coffee, alcohol and risk of coronary heart disease among Japanese men living in Hawaii. N Engl J Med 1977;297:405-9.

[48] Heyden S, Tyroler HA, Heiss G, Hames CG, Bartel A. Coffee consumption and mortality. Arch Intern Med 1978;138: $1472-5$.

[49] Murray SS, Bjelke E, Gibson RW, Schuman LM. Coffee consumption and mortality from ischemic heart disease and other causes: results from the Lutheran Brotherhood Study, 1966-1978. Am J Epidemiol 1981;113:661-7.

[50] Woodward M, Tunstall-Pedoe H. Coffee and tea consumption in the Scottish Heart Health Study follow-up: conflicting relations with coronary risk factors, coronary heart disease, and all cause mortality. J Epidemiol Community Health 1999;53:481-7.

[51] Tibblin G, Wilhelmsen L, Werko L. Risk factors for myocardial infarction and death due to ischemic heart disease and other causes. Am J Cardiol 1975;35:514-22.

[52] Heyden S, Tyroler HA, Cassel JC, Hames CG, Becker C, Heiss G. Coffee consumption and mortality in a community study. Z Ernahrungswiss 1976;15:143-50.

[53] Wilson PWF, Garrison RJ, Kannel WB, McGee DL, Castelli WP. Is coffee consumption a contributor to cardiovascular disease? Arch Intern Med 1989;149:1169-72.

[54] Gartside PS, Glueck CJ. Relationship of dietary intake to hospital admission for coronary heart and vascular disease: the NHANES II national probability study. J Am Coll Nutr 1993;12:676-84.

[55] Rosengren A, Wilhelmsen L. Coffee, coronary heart disease and mortality in middle-aged Swedish men: findings from the Primary Prevention Study. J Intern Med 1991; 230:67-71. 
[56] Mukamal KJ, Maclure M, Muller JE, Sherwood JB, Mittleman MA. Caffeinated coffee consumption and mortality after acute myocardial infarction. Am Heart J 2004;147:999-1004.

[57] Heyden S, Heyden F, Heiss G, Hames CG. Smoking and coffee consumption in three groups: cancer deaths, cardiovascular deaths and living controls, a prospective study in Evans County, Georgia. J Chron Dis 1979;32:673-7.

[58] Puccio EM, McPhillips JB, Barrett-Connor E, Ganiats TG. Clustering of atherogenic behaviours in coffee drinkers. Am J Public Health 1990;80:1310-3.

[59] Jacobsen BK, Thelle DS. The Tromso Heart Study: is coffee drinking an indicator of a life style with high risk of ischemic heart disease? Acta Med Scand 1987;222:215-21.

[60] Stanton MF, Ahrens RA, Douglass LW. Coffee and cola beverage consumption as heart disease risk factors in men. Experientia 1978;34:1882-3.

[61] Jazbec A, Simic D, Corovic N, Durakovic Z, Pavlovic M. Impact of coffee and other selected factors on general mortality and mortality due to cardiovascular disease in Croatia. J Health Popul Nutr 2003;21:332-40.

[62] Hakim AA, Ross GW, Curb JD, Rodriguez BL, Burchfield CM, Sharp DS, et al. Coffee consumption in hypertensive men in older middle-aged and the risk of stroke: the Honolulu Heart Program. J Clin Epidemiol 1998;51:487-94.

[63] Happonen P, Voutilainen S, Salonen JT. Coffee drinking is dose-dependently related to the risk of acute coronary events in middle-aged men. J Nutr 2004;134:2381-6.

[64] Kleemola $P$, Jousilahti $P$, Pietinen $P$, Vartiainen $E$, Tuomilehto J. Coffee consumption and the risk of coronary heart disease and death. Arch Intern Med 2000;160: $3393-400$.
[65] Egger M, Davey-Smith G, Schneider M, Minder C. Bias in meta-analysis detected by a simple, graphical test. BMJ 1997;315:629-34.

[66] Rosenberg MS. The file-drawer problem revisited: a general weighted method for calculating fail-safe numbers in metaanalysis. Evolution Int J Org Evolution 2005;59:464-8.

[67] Klag MJ, Wang N-Y, Meoni LA, Brancati FL, Cooper LA, Liang $\mathrm{K}-\mathrm{Y}$, et al. Coffee intake and risk of hypertension. The Johns Hopkins Precursors Study. Arch Intern Med 2002;162:657-62.

[68] Winkelmayer WC, Stampfer MJ, Willett WC, Curhan GC. Habitual caffeine intake and the risk of hypertension in women. JAMA 2005;294:2330-5.

[69] Zampelas A, Panagiotakos DB, Pitsavos C, Chrysohoou C, Stefanadis C. Association between coffee consumption and inflammatory markers in healthy persons: the ATTICA study. Am J Clin Nutr 2004;80:862-7.

[70] Urgert R, van Vliet T, Zock PL, Katan MB. Heavy coffee consumption and plasma homocysteine: a randomized controlled trial in healthy volunteers. Am J Clin Nutr 2000; 72:1107-10.

[71] van Dam RM. Coffee and type 2 diabetes: from beans to beta-cells. Nutr Metab Cardiovasc Dis 2006;16:69-77.

[72] Ruhl CE, Everhart JE. Coffee and caffeine consumption reduce the risk of elevated serum alanine aminotransferase activity in the United States. Gastroenterology 2005;128: 24-32.

[73] McCusker RR, Goldberger BA, Cone EJ. Caffeine content of specialty coffees. J Anal Toxicol 2003;27:520-2.

[74] Cornelis MC, El-Sohemy A, Kabagambe EK, Campos H. Coffee, CYP1A2 genotype, and risk of myocardial infarction. JAMA 2006;295:1135-41.

Available online at www.sciencedirect.com

\section{$\because$ ScienceDirect}

